\title{
How can we measure endometriosis-associated pelvic pain?
}

\author{
Christoph Gerlinger ${ }^{1}$, Ulrike Schumacher ${ }^{2}$, René Wentzeck ${ }^{1}$, Kerstin Uhl-Hochgräber ${ }^{3}$, \\ Erich F. Solomayer ${ }^{4}$, Heinz Schmitz ${ }^{5}$, Thomas Faustmann ${ }^{6}$, Christian Seitz ${ }^{5}$ \\ ${ }^{1}$ Bayer HealthCare, Global Biostatistics, Berlin - Germany \\ 2Jenapharm and Zentrum für Klinische Studien, Universitätsklinikum Jena, Jena - Germany \\ ${ }^{3}$ Bayer HealthCare, Global Health Economics and Outcomes Research, Berlin - Germany \\ ${ }^{4}$ University of Saarland, Department of Obstetrics, Gynecology, and Reproductive Medicine, Homburg/Saar - Germany \\ ${ }^{5}$ Bayer HealthCare, Global Clinical Development, Berlin - Germany \\ ${ }^{6}$ Bayer HealthCare, Global Medical Affairs Women's Healthcare, Berlin - Germany
}

\begin{abstract}
Purpose: The aim of our work was to explore which of the most commonly used pain scales is best suited to assess treatment success in endometriosis therapy and, therefore, qualifies best to be used as primary endpoint for clinical studies in this indication.

Methods: We compared patient's responses on the different pain scales Visual Analog Scale, Biberoglu and Behrman Score, and SF-36 Bodily Pain Subscale with the Clinical Global Impression score. Parametric and non-parametric correlation coefficients and effect sizes were calculated.

Results: A total of 428 patients with endometriosis-associated pelvic pain from three studies were included in our analyses. Their mean age was $31.4 \pm 6.3$ years and their mean pain score on the visual analog scale was $58.1 \pm 21.9$ at baseline. The highest correlation with the Clinical Global Impression score was observed for the visual analog scale followed by the B\&B pelvic pain item. The highest effect sizes were found for dysmenorrhea and SF-36 bodily pain subscale followed by the visual analog scale.

Conclusions: A general measure of endometriosis-related pain can be recommended as primary endpoint in clinical trials to assess painful symptoms of endometriosis. In addition, a disease-specific quality of life tool is recommended to help interpret impact on patients' daily activities.
\end{abstract}

KeY wORDs: Effect size, Endometriosis, Pelvic pain, Visual analogue scale

Accepted: September 4, 2012

\section{INTRODUCTION}

Pain is commonly reported as the most relevant symptom for endometriosis patients $(1,2)$ and most women with symptomatic endometriosis experience some symptoms of pain associated with their menstrual cycle (3). The ways to measure pain symptoms in clinical trials differ substantially: pain scales used in past and recent endometriosis trials include, among others, the Biberoglu \& Behrman Score (B\&B) (4), the Visual Analog Scale (VAS) (5), and the Numerical Rating Scale (NRS) (6). This problem was recently addressed by an expert group who gave recommendations regarding endpoints to be included in clinical trials for endometriosis (7). The varied nature limits direct comparison of the trial results and makes meta-analyses difficult. The situation is further complicated by the differ- 
ent preferences of important regulatory agencies. While the VAS is one of the most widely used pain scales in Europe and was also used as a primary endpoint in studies of the most recently approved drug for treatment of endometriosis in Europe (8), the FDA indicated a clear preference for the B\&B score and its components (9). In Japan the most recent approval for an endometriosis therapy was based on studies using several five-point pain measurements and a VAS (10). While these pain scales assess the most relevant clinical symptom of endometriosis they may not cover other important aspects of the disease. Therefore, more comprehensive tools to assess quality of life (QoL) are often included in endometriosis clinical trials $(5,11)$, e.g. the SF-36 (12). This nondisease-specific QoL instrument has recently been validated for use in patients with endometriosis (13). A subscale to assess "bodily pain" is part of this instrument showing a good correlation with other pain scales in endometriosis patients. Nondisease-specific tools such as the SF-36 facilitate comparison of the burden of the disease with other illnesses. Disease-specific tools such as the EHP-30 or -5 are generally more sensitive, but do not facilitate comparison across diseases.

The aim of our work was to explore which of the most commonly used pain scales is most suitable to assess treatment success in endometriosis therapy and, therefore, best qualifies for use as a primary endpoint in clinical studies for this indication. To this end, we compared different pain scales with the Clinical Global Impression (CGI) score. This single item tool assesses "overall treatment success" without differentiation into specific symptoms or aspects of QoL. It qualifies perfectly as a so- called "anchor", i.e. a very simple tool to compare the more complex tools against (14).

Specifically, we wanted to analyze (A) which pain scale reflects patient satisfaction best and (B) which instrument is most suitable to assess treatment effects.

\section{MATERIALS AND METHODS}

\section{Studies}

Three recent studies with a similar design used measurement of endometriosis-associated pelvic pain (EAPP) on a VAS as primary endpoint (Fig. 1); several other patient reported outcomes (PROs) were assessed as secondary endpoints. Study 1 (5) was a multicenter, open-label, controlled, randomized, parallel

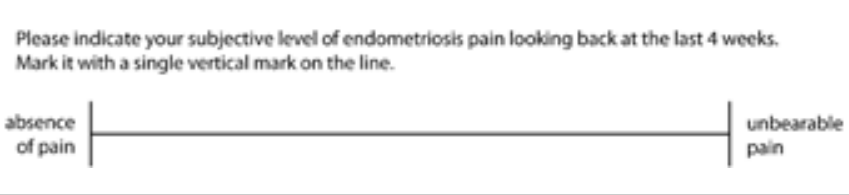

Fig. 1 - Visual analogue scale.

group study to investigate the efficacy and safety of daily oral administration of $2 \mathrm{mg}$ Dienogest (DNG) versus intramuscular administration of $3.75 \mathrm{mg}$ Leuprorelin Acetate (LA) every four weeks for the treatment of symptomatic endometriosis over 24 weeks. Study 2 (11) was a multicenter, double-blind, randomized, placebo-controlled, parallel-group study to investigate the efficacy and safety of daily oral administration of $2 \mathrm{mg}$ DNG tablets for the treatment of endometriosis over 12 weeks. Study 3 (ClinicalTrials.gov Identifier: NCT00185341) compared an experimental treatment $(E T)$ to placebo $(\mathrm{PL})$ and used a design almost identical to study 2. ET in study 3 was revealed to be no more efficacious than PL. For the purpose of this analysis, ET was therefore pooled with PL. Patients on LA were excluded from our analyses because of the expected confounding of the QoL scores with the typical side effects of LA, such as hot flushes and amenorrhea, which precluded the pooling of the LA-treated patients with those of the other groups.

The study protocols were approved by local independent Ethics Committees and all participants provided written informed consent before study enrolment. Studies were performed in accordance with the amended version of the Declaration of Helsinki and complied with Good Clinical Practice.

\section{Patients}

In all studies, 18 to 45 -year-old women between menarche and menopause, and in good general health except for endometriosis, were eligible for inclusion. Inclusion criteria included laparoscopically confirmed endometriosis (stage I-IV, using revised American Society of Reproductive Medicine [r-ASRM] scoring) $(15,16)$, which was determined at laparoscopy within 12 months prior to study baseline. Patients had to report at screening and baseline an EAPP score of at least $30 \mathrm{~mm}$ (study 2) or $40 \mathrm{~mm}$ (study 3) on a VAS, where $0 \mathrm{~mm}$ represents absence of pain and $100 \mathrm{~mm}$ indicates unbearable pain. For study 1 there was no minimal VAS score requirement at study entry.

Exclusion criteria included pregnancy or breast feeding, use of an intrauterine device, amenorrhea within three months of screening, signs or symptoms of therapy resistant endometriosis or need for immediate surgical treatment of endometriosis, 


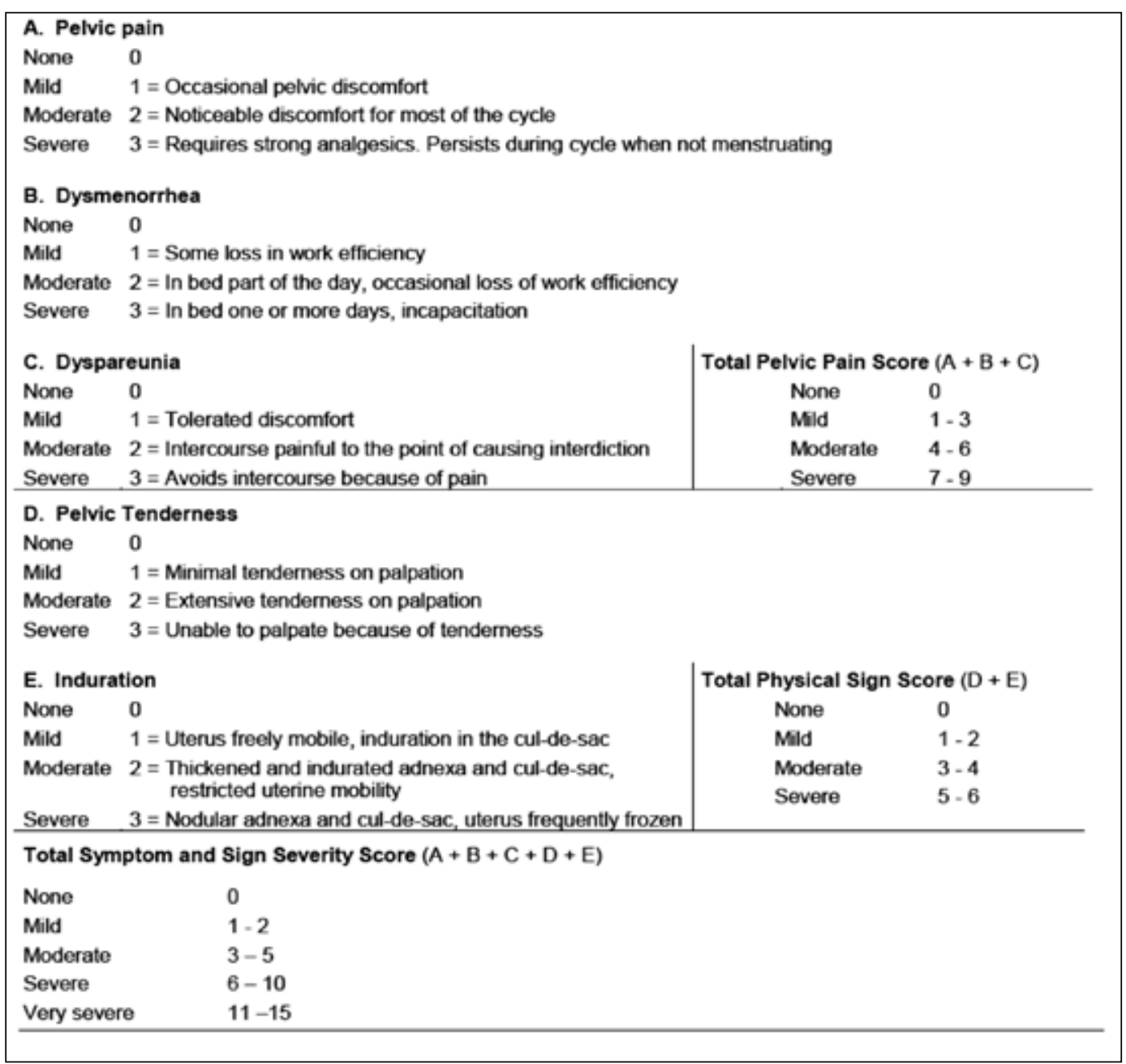

Fig. 2 - Biberoglu and Behrman score. recent use of hormonal agents (e.g., GnRH agonists $\leq 6$ months before screening, progestins or danazol $\leq 3$ months before screening, or oral contraceptives $\leq 1$ month before screening), clinically relevant findings at gynecologic examination other than endometriosis, or an abnormal cervical cytologic smear in the last three months.

\section{Endpoints}

Among other clinical parameters not covered in this paper, the following endpoints were documented in all three studies and were used for this pooled analysis.

The VAS for EAPP (Fig. 1) is a $10 \mathrm{~cm}$ long horizontal line with its extremes marked as "absence of pain" and "unbearable pain". The patient ticks her pain level on the line and the distance from the left extreme "absence of pain" to the tick mark is measured in millimeters yielding a pain score from 0 to 100 .

The B\&B score (4) consists of a rating $(0=$ none; $1=$ mild; $2=$ moderate; 3 = severe) based on the patient's assessment of three distinct pain symptoms (dysmenorrhea, pelvic pain, dyspareunea) and on two findings obtained during gynecologic palpation (tenderness, induration). The three pain symptoms are combined with the "pelvic symptoms score" and the two findings with the "physical symptoms score". Finally, both can be combined with the "B\&B total sum score" (Fig. 2).

The CGI scale (14) originally consists of three items: severity of illness, efficacy index, and global improvement each answered on a seven-point scale. The studies reported here used a patient-reported modification of the global improvement item (1 = Very much satisfied; 2 = Much satisfied; 3 = Minimally satisfied; 4 = Neither satisfied nor dissatisfied; $5=$ Minimally dissatisfied; $6=$ Much dissatisfied; and $7=$ Very much dissatisfied).

The SF-36 (12) can be considered the gold standard for QoL measurements under different conditions, but it is not specific for endometriosis. It includes the assessment of 
symptoms that may disrupt working ability, social relationships or sexual functioning. The SF-36 consists of 36 items combined into the eight subscales Role-Emotional, Vitality, General Health, Mental Health, Bodily Pain, Physical Functioning, Role Physical, and Social Functioning. These are further combined into the two summary measures Mental Health and Physical Health. The scoring system of the SF36 assigns higher numeric scores to a better health status.

\section{Statistical analysis}

Efficacy and quality of life data were pooled from the three individual studies into one data set for statistical analyses. Following the "intention to treat" principle, all patients that provided data were included in the analyses, regardless of possible protocol deviations.

Change from baseline was analyzed for all variables except for the CGI which is already a measure of change. For the assessment of correlations of pain scales with the CGI the difference in score of a pain scale at each time point (12 or 24 weeks, respectively) versus baseline was compared to the CGI at the same time point. For comparison of effect sizes only data after 12 weeks of treatment were utilized because the treatment effect can be expected to change over time and the PL groups were only treated for 12 weeks. For the B\&B score only the patient reported outcomes dysmenorrhea, pelvic pain, and dyspareunea were analyzed. In addition, the "pelvic symptoms score", a summary measure of dysmenorrhea, pelvic pain, and dyspareunea was analyzed. In the same way, the analyses of the SF-36 focused on bodily pain subscale.

Variables were analyzed by descriptive statistics using version 9.2 of SAS software (SAS, Cary, NC, USA www.sas. com). Parametric, i.e. Pearson $\left(r_{p}\right)$, and nonparametric, i.e. Spearman $\left(r_{s}\right)$, correlation coefficients were calculated for the whole population as well as separately by treatment assignment. Effect sizes were calculated as the mean of the change from baseline divided by the standard deviation of the change.

\section{RESULTS}

A total of 428 patients, 222 receiving active treatment (i.e. $\mathrm{DNG}$ ) and 206 receiving inactive treatment (ET or $\mathrm{PL}$ ), were included in our analyses. Most patients (98.1\%) were Cau- casian as all three studies were performed in Europe. Mean patient age was 31.4 years $( \pm 6.3)$. At baseline, patients reported substantial mean levels of pain symptoms on the respective scales: $58.1( \pm 21.9)$ on the VAS, $4.8( \pm 1.7)$ for the B\&B subscore on pelvic symptoms, and $42.3( \pm 20.0)$ on the bodily pain subscale of the SF-36. There was no relevant difference in any of the baseline parameters between the treatment groups, see Table I.

The correlations of the different scales with regard to changes from baseline to week 12 are summarized in Table II. Almost all comparisons showed a correlation in the expected direction, i.e. improvement of pain assessed by one scale was generally positively correlated with improvement of pain assessed by the other scales. It is important to mention that the scoring system of the SF-36 assigns higher numeric scores to a better health status whereas all other instruments assign lower numeric values to less pain. Based on the Pearson coefficient, the highest correlation with the CGI efficacy index was observed for the VAS $\left(r_{\mathrm{p}}=0.53\right)$ followed by the $B \& B$ pelvic pain item $\left(r_{p}=0.40\right)$ while the $B \& B$ dyspareunia item did not show a relevant correlation with the CGI efficacy index $\left(r_{p}=0.19\right)$. Most scales showed a moderate correlation between themselves; again with the exception of the $B \& B$ dyspareunia item which was not relevantly correlated with either B\&B dysmenorrhea $\left(r_{p}=0.24\right)$ or SF-36 bodily pain $\left(r_{p}=-0.15\right)$. The non-parametric Spearman correlations were similar to the Pearson correlations for all variables except for SF-36 bodily pain, where $r_{p}$ indicated a substantially stronger correlation than the $r_{S}$. Based on $r_{S}$ there was no relevant correlation between SF-36 bodily pain and any of the other pain scales $\left(r_{s}=0.03\right.$ to -0.13$)$.

The results presented in Table II are regardless of the treatment. When data were analyzed by treatment, the structure and magnitude of the correlations were not substantially altered (data not shown).

Effect sizes of the different variables investigated are shown in Table III for both, comparison of end-of-study versus baseline and comparison between treatment groups. Effect sizes for comparison between treatment groups ranged from 0.24 (B\&B dyspareunia) to 0.709 (B\&B dysmenorrhea). The B\&B dysmenorrhea item, B\&B pelvic symptoms score, SF36 bodily pain, and VAS had the largest effect sizes regarding the comparisons within each treatment group as well as between active and in-active groups. For the comparison between treatment groups the effect sizes of the B\&B dysmenorrhea item, B\&B pelvic symptoms score, and VAS were $0.709,0.630$, and 0.703 for SF-36 bodily pain, respectively. 
TABLE I - DEMOGRAPHICS AND BASELINE SCORES

\begin{tabular}{|c|c|c|c|c|c|c|}
\hline & \multicolumn{2}{|c|}{ Active } & \multicolumn{2}{|c|}{ Inactive } & \multicolumn{2}{|c|}{ Total } \\
\hline & $\mathrm{n}$ & $\%$ & $\mathrm{n}$ & $\%$ & $\mathrm{n}$ & $\%$ \\
\hline Total Number of patients & 222 & 100 & 206 & 100 & 428 & 100 \\
\hline \multirow[t]{2}{*}{ Ethnic group: Caucasian } & 217 & 97.7 & 203 & 98.5 & 420 & 98.1 \\
\hline & Mean & SD & Mean & SD & Mean & SD \\
\hline Age (years) & 31.0 & 6.4 & 31.9 & 6.1 & 31.4 & 6.3 \\
\hline Weight (kg) & 62.44 & 10.52 & 63.15 & 10.89 & 62.78 & 10.69 \\
\hline \multirow[t]{2}{*}{ Body Mass Index $\left(\mathrm{kg} / \mathrm{m}^{2}\right)$} & 22.66 & 3.40 & 22.86 & 3.71 & 22.75 & 3.55 \\
\hline & Mean & SD & Mean & SD & Mean & SD \\
\hline Visual Analogue Scale & 54.93 & 24.62 & 61.52 & 17.93 & 58.11 & 21.87 \\
\hline B\&B score Pelvic pain & 1.8 & 0.8 & 1.7 & 0.7 & 1.8 & 0.7 \\
\hline B\&B score Dysmenorrhea & 1.8 & 0.8 & 1.8 & 0.8 & 1.8 & 0.8 \\
\hline B\&B score Dyspareunia & 1.2 & 0.9 & 1.2 & 0.9 & 1.2 & 0.9 \\
\hline B\&B score Induration & 1.0 & 0.8 & 1.1 & 0.8 & 1.1 & 0.8 \\
\hline B\&B score Pelvic tenderness & 1.3 & 0.7 & 1.5 & 0.7 & 1.4 & 0.7 \\
\hline B\&B subscore Sum of pelvic symptoms & 4.8 & 1.7 & 4.8 & 1.8 & 4.8 & 1.7 \\
\hline B\&B subscore Sum of physical signs & 2.4 & 1.2 & 2.6 & 1.2 & 2.5 & 1.2 \\
\hline B\&B Sum of scores & 7.2 & 2.5 & 7.4 & 2.5 & 7.3 & 2.5 \\
\hline SF-36 subscale Emotional Role function & 57.80 & 40.10 & 63.50 & 41.00 & 59.52 & 40.37 \\
\hline SF-36 subscale Energy / Vitality & 47.20 & 19.20 & 49.60 & 18.50 & 47.90 & 19.00 \\
\hline SF-36 subscale General Health & 51.50 & 21.70 & 46.30 & 20.10 & 49.90 & 21.30 \\
\hline SF-36 subscale Mental health & 57.70 & 19.20 & 57.80 & 18.90 & 57.71 & 19.04 \\
\hline SF-36 subscale Bodily Pain & 41.00 & 21.50 & 45.30 & 15.60 & 42.30 & 20.00 \\
\hline SF-36 subscale Physical function & 77.90 & 20.00 & 78.40 & 18.00 & 78.03 & 19.41 \\
\hline SF-36 subscale Physical role function & 49.20 & 38.90 & 55.30 & 37.90 & 51.00 & 38.60 \\
\hline SF-36 subscale Social function & 64.60 & 24.10 & 65.00 & 21.80 & 64.75 & 23.39 \\
\hline SF-36 summary measure Physical Health & 49.98 & 8.76 & 49.99 & 8.76 & 50.00 & 8.72 \\
\hline SF-36 summary measure Mental Health & 49.90 & 10.25 & 50.01 & 10.33 & 49.94 & 10.30 \\
\hline
\end{tabular}

\section{DISCUSSION}

The pain and QoL scales investigated in this study are used to measure endometriosis-associated pain in clinical studies. In our study all scales showed the expected direction of correlation with the CGI efficacy index which was used as the anchor indicating overall treatment success in this analysis. However, the different scales showed substantial variability in their degree of correlation with the anchor tool and their ability to detect treatment effects.

The superior correlation of the VAS with overall treatment satisfaction may result from the quite general wording of the question used, which allows a patient to consider a wide range of subjective and individual symptoms relating to this item. As a result this may be a better tool to assess overall treatment satisfaction than more specific questions. Another possible interpretation of this finding may be that patients care most about the overall amount of pain while the type of pain or subtle differences in the way it is worded may be less important. Nevertheless, differentiation between specific types of pain may be useful as secondary endpoints in clinical trials, e.g. to help understand the mode of action of a specific treatment.

The VAS, as the best instrument in this respect, still ac- 
TABLE II - CORRELATIONS BETWEEN THE CHANGE FROM BASELINE BETWEEN DIFFERENT PAIN SCORES

\begin{tabular}{|c|c|c|c|c|c|c|c|}
\hline $\begin{array}{l}\text { Pearson } \\
\text { Spearman }\end{array}$ & $\begin{array}{l}\text { CGI, efficacy } \\
\text { index }\end{array}$ & VAS & $\begin{array}{c}\text { B\&B } \\
\text { dysmenorrhea }\end{array}$ & $\begin{array}{c}\text { B\&B } \\
\text { dyspareunia }\end{array}$ & $\begin{array}{c}\text { B\&B } \\
\text { pelvic } \\
\text { pain }\end{array}$ & $\begin{array}{c}\text { B\&B } \\
\text { subscore } \\
\text { pelvic } \\
\text { symptoms }\end{array}$ & $\begin{array}{c}\text { SF-36 } \\
\text { bodily } \\
\text { pain }\end{array}$ \\
\hline CGI, efficacy index & & 0.53 & 0.35 & 0.19 & 0.40 & 0.39 & -0.40 \\
\hline VAS & 0.56 & & 0.47 & 0.15 & 0.40 & 0.47 & -0.50 \\
\hline B\&B dysmenorrhea & 0.37 & 0.51 & & 0.24 & 0.38 & 0.78 & -0.47 \\
\hline B\&B dyspareunia & 0.17 & 0.11 & 0.16 & & 0.27 & 0.67 & -0.15 \\
\hline B\&B pelvic pain & 0.40 & 0.36 & 0.31 & 0.28 & & 0.73 & -0.32 \\
\hline B\&B subscore pelvic symptoms & 0.40 & 0.49 & 0.76 & 0.62 & 0.70 & & -0.46 \\
\hline SF-36 bodily pain & -0.13 & -0.12 & 0.03 & 0.01 & -0.12 & -0.06 & \\
\hline
\end{tabular}

CGI, clinical global impression; VAS, visual analog scale

TABLE III - EFFECT SIZES FOR CHANGES FROM BASELINE

\begin{tabular}{|c|c|c|c|c|c|}
\hline Variable & Treatment & $\mathbf{n}$ & Mean & SD & Effect size \\
\hline \multirow[t]{3}{*}{ Visual Analog Scale } & Active Treatment & 210 & -31.890 & 30.340 & -1.051 \\
\hline & Inactive Treatment & 185 & -19.330 & 21.128 & -0.915 \\
\hline & Active - Inactive & 395 & -12.561 & 26.363 & -0.476 \\
\hline \multirow[t]{3}{*}{ B\&B dysmenorrhea } & Active Treatment & 214 & -1.252 & 1.101 & -1.137 \\
\hline & Inactive Treatment & 194 & -0.552 & 0.852 & -0.648 \\
\hline & Active - Inactive & 408 & -0.701 & 0.988 & -0.709 \\
\hline \multirow[t]{3}{*}{ B\&B dyspareunea } & Active Treatment & 190 & -0.611 & 0.906 & -0.674 \\
\hline & Inactive Treatment & 166 & -0.410 & 0.755 & -0.542 \\
\hline & Active - Inactive & 356 & -0.201 & 0.837 & -0.240 \\
\hline \multirow[t]{3}{*}{ B\&B pelvic pain } & Active Treatment & 214 & -0.907 & 0.834 & -1.087 \\
\hline & Inactive Treatment & 194 & -0.552 & 0.802 & -0.688 \\
\hline & Active - Inactive & 408 & -0.355 & 0.817 & -0.435 \\
\hline \multirow[t]{3}{*}{ B\&B pelvic symptoms } & Active Treatment & 190 & -2.732 & 2.010 & -1.359 \\
\hline & Inactive Treatment & 166 & -1.554 & 1.707 & -0.910 \\
\hline & Active - Inactive & 356 & -1.177 & 1.870 & -0.630 \\
\hline \multirow[t]{3}{*}{ SF-36 bodily pain } & Active Treatment & 212 & 28.245 & 27.523 & 1.026 \\
\hline & Inactive Treatment & 91 & 10.286 & 20.499 & 0.502 \\
\hline & Active - Inactive & 303 & 17.960 & 25.540 & 0.703 \\
\hline
\end{tabular}

SD, standard deviation

counts for only $28 \%$ of the variability in patient's satisfaction with treatment (coefficient of determination $\mathrm{R}^{2}$ $=0.28$ ). In contrast, the SF-36 only accounts for $16 \%$ of the variability. This strongly supports the need to include validated disease-specific QoL tools to assess aspects of endometriosis other than pain. An example of such a tool is the EHP-30 and its short-form the EHP-5. Other tools are currently being developed following the recently published FDA guidance on development of PRO instruments (16 and VALEPRO study, ClinicalTrials.gov Identifier: NCT01643122). However, these tools were not available at the time the reported trials were performed. 
Ethical and financial requirements of clinical trials are to achieve a valid result while exposing as few patients as possible to an experimental treatment. The endpoint with the highest effect size requires the smallest sample size. Given the effect sizes calculated in this analysis, a clinical trial using the bodily pain subscale of the SF-36 would require a sample size of 88 patients compared to 188 patients when the VAS is used to attain the same statistical power of $90 \%$. The dysmenorrhea item of the B\&B showed an even better effect size. This result, however, may be influenced by a high proportion of amenorrhea caused by active treatment in many patients and may not hold true in other types of treatment based on other modes of action. This study did not assess the aspect of content validity of the different scales for their use in endometriosis patients. This aspect, however, is crucial according to guidance issued by the FDA recently (17). The widely used B\&B scale especially does not appear to fulfill the requirements for state-of-the-art PRO tools as its items were developed based on expert opinion rather than patient input. The same holds true for the VAS but may be less critical as the question is phrased so generally that patients will probably include a wide range of symptoms. In addition, the VAS appears to be the only scale with an empirically established minimal clinically important difference (MCID). Gerlinger et al. (18) found the MCID for the VAS scale in endometriosis patients to be in the order of $10 \mathrm{~mm}$. For other endpoints no comparable empirically derived MCID is available. FDA and clinical experts recommended using a numeric rating scale (NRS) instead of a VAS which can be expected to provide similar results (19).

The strengths of this study include its large sample size and the possibility for direct comparison of several scales within the same group of patients. This meta-analysis substantially increases the level of evidence while supporting expert recommendations expressed earlier on the same topic (20) and gives practical guidance to clinical investigators in the field of endometriosis.

Limitations include the relatively short observation period (12 weeks) which may not provide the full picture on the effect sizes and the coverage of only few distinct treatment options. The PRO instruments investigated in this study were applied relatively infrequently. This contrasts with an increasing tendency from Regulatory Agencies to request documentation of data on pain on a daily level by using electronic diaries for example. Limitations also include the fact that the analysis is based on pooled data from three studies which may introduce a higher degree of variability compared to data obtained from one study.

\section{Summary and recommendation}

Based on our analysis, a general measure of endometriosis-related pain (VAS/NRS) best reflects patient's overall satisfaction with treatment and can be recommended as primary endpoint in clinical trials to assess painful symptoms of endometriosis. Daily electronic assessment together with a bleeding diary would enable differentiating between pain related or not related to bleeding without the risk of narrowing down the scope of the question. In addition, a disease-specific QoL instrument is recommended in order to allow interpretation of the impact on patient's daily activities. Potentially, this needs to be applied less frequently than questions on pain.

Financial support: This study was sponsored by Bayer Healthcare.

Conflict of interest: CG RW KUH HS TF and CS are employees of Bayer. US is an employee of Jenapharm, a subsidiary of Bayer.

Informed consent: All patients gave informed consent prior to inclusion into the studies.

Address for correspondence

Dr. Christoph Gerlinger

Bayer HealthCare

Müllerstraße 178

13342 Berlin, Germany

christoph.gerlinger@bayer.com

\section{REFERENCES}

1. Kennedy S, Bergqvist A, Chapron C, et al. ESHRE guideline for the diagnosis and treatment of endometriosis. Hum Reprod 2005; 20: 2698-704.

2. Practice Committee of American Society for Reproductive Medicine. Treatment of pelvic pain associated with endometriosis. Fertil Steril 2008; 90: S260-9.

3. Bernuit D EA, Halis G, Strothmann A, Gerlinger C, Geppert $\mathrm{K}$, Faustmann T. Female perspectives on endometriosis: findings from the uterine bleeding and pain women's research study. J Endometr 2011; 3: 73-85. 
4. Biberoglu KO, Behrman SJ. Dosage aspects of danazol therapy in endometriosis: short-term and long-term effectiveness. Am J Obstet Gynecol 1981 15; 139: 645-54.

5. Strowitzki T, Marr J, Gerlinger C, Faustmann T, Seitz C. Dienogest is as effective as leuprolide acetate in treating the painful symptoms of endometriosis: a 24-week, randomized, multicentre, open-label trial. Hum Reprod 2010; 25: 633-41.

6. Guzick DS, Huang LS, Broadman BA, Nealon M, Hornstein MD. Randomized trial of leuprolide versus continuous oral contraceptives in the treatment of endometriosis-associated pelvic pain. Fertil Steril 2011; 95: 1568-73.

7. Vincent K, Kennedy S, Stratton P. Pain scoring in endometriosis: entry criteria and outcome measures for clinical trials. Report from the Art and Science of Endometriosis meeting. Fertil Steril 2010; 93: 62-7.

8. Committee for Proprietary Medicinal Products (CPMP). Note for Guidance on Clinical Investigation of Medicinal Products for treatment of nociceptive pain (CPMP/EWP/612/00). London: EMEA, 2002.

9. Food and Drug Administration CfDEaR. Statistical Review(s). Depo-subQ provera 104TM. Medroxyprogesterone acetate injectable suspension $104 \mathrm{mg} / 0.65 \mathrm{~mL}$. 2005.

10. Harada T, Momoeda M, Taketani $Y$, et al. Dienogest is as effective as intranasal buserelin acetate for the relief of pain symptoms associated with endometriosis--a randomized, double-blind, multicenter, controlled trial. Fertil Steril 2009; 91: 675-81.

11. Strowitzki T, Faustmann T, Gerlinger C, Seitz C. Dienogest in the treatment of endometriosis-associated pelvic pain: a 12week, randomized, double-blind, placebo-controlled study. Eur J Obstet Gynecol Reprod Biol 2010; 151: 193-8.
12. Ware JE KM. SF-36 Physical and Mental Health Summary Scales: A Manual for Users of Version 1. 2nd edn. Lincoln, RI, USA: QualityMetric Incorporated; 2001.

13. Stull D WR, Kreif N, Colligs A, Seitz C, Gerlinger C. Validation of the SF-36 in patients with endometriosis. Value Health 2009; 12: A295.

14. Guy W. Clinical Global Impressions. In: (ADM) DP, editor. ECDEU Assessment Manual for Psychopharmacology. Rockville, MD: National Institute for Mental Health 1976; 218-22.

15. Revised American Fertility Society classification of endometriosis: 1985. Fertil Steril 1985; 43: 351-2.

16. Revised American Society for Reproductive Medicine classification of endometriosis: 1996. Fertil Steril 1997; 67: 817-21.

17. U.S. Department of Health and Human Services Food and Drug Administration Center for Drug Evaluation and Research Center for Biologics Evaluation and Research Center for Devices and Radiological Health. Guidance for Industry. Patient-reported Outcome Measures: Use in Medical Product Development to Support Labeling Claims. Rockville, MD2009.

18. Gerlinger C, Schumacher U, Faustmann T, Colligs A, Schmitz $\mathrm{H}$, Seitz C. Defining a minimal clinically important difference for endometriosis-associated pelvic pain measured on a visual analog scale: analyses of two placebo-controlled, randomized trials. Health Qual Life Outcomes 2010; 8: 138.

19. Ahlers SJ, van Gulik L, van der Veen AM, et al. Comparison of different pain scoring systems in critically ill patients in a general ICU. Crit Care 2008;12(1):R15.

20. Vincent K. Pelvic pain in women: clinical and scientific aspects. Curr Opin Support Palliat Care 2011; 5: 143-9. 